\section{Integrative Medicine \\ International}

\title{
The Molecular Mechanism of Diastolic Heart Failure
}

\author{
Jialin Gu Feiran Zhao Yuqi Wang Junjie Gao Xiaolong Wang \\ Jingui Xue Hua Zhou \\ Department of Cardiovascular Medicine, Shuguang Hospital Affiliated to Shanghai \\ University of TCM, Shanghai, China
}

\section{Key Words}

Diastolic heart failure · Molecular mechanisms · Aldosterone $\cdot$ Myocardial remodeling ·

Inflammatory cytokines · Myocardial calcium cycling

\begin{abstract}
Diastolic heart failure (DHF) is a group of clinical syndromes related to the performance of the pulmonary circulation and systemic circulation, with normal left ventricular (LV) systolic function. The pathophysiology of diastolic dysfunction includes delayed relaxation, impaired LV filling, and/or increased stiffness. These conditions result in impaired LV diastolic relaxation ability and a decrease in myocardial compliance. In recent years, studies on the mechanisms of DHF have focused on the renin-angiotensin-aldosterone system, inflammatory cytokines, oxidative stress, the process of myocardial calcium cycling, and associated proteins. The pathomechanism has been proven to be due to a deficiency in ATP, and $\mathrm{Ca}^{2+}$ cannot be reduced by sarcoplasmic reticulum calcium pump (SERCA2a), which leads to myocardial diastolic dysfunction. The correlation between the degradation process of ATP and its metabolites and DHF has also been studied in recent years. This paper summarizes the views on the above and analyzes the correlations between the molecular mechanisms.
\end{abstract}

(C) 2015 The Author(s)

Published by S. Karger AG, Basel

\section{Aldosterone and Myocardial Remodeling}

Overactivation of the renin-angiotensin-aldosterone system (RAAS) in conjunction with myocardial fibrosis is currently believed to be one of the mechanisms that lead to the development of diastolic heart failure (DHF) [1]. In the RAAS, aldosterone is the main substance 
which causes myocardial remodeling. Aldosterone can also increase NADPH oxidase, activate nuclear factor- $\kappa \mathrm{B}$, and increase the expression of matrix metalloproteinase (MMP) 2 and mRNA of type I collagen and GTGF. Type I collagen is a fiber. Excessive collagen accumulation increases the tension of the stromal cells while weakening their extensibility and elasticity, which leads to myocardial stiffness [2]. In contrast, in systolic heart failure, myocardial interstitium is mainly composed of type III collagen. Due to its greater extensibility and elasticity, the chambers of the heart increase but myocardial stiffness does not drastically change [2,3]. Aldosterone can also be produced by oxidative stress mediated by myocardial remodeling. Possible mechanisms are as follows: (1) aldosterone upregulates the expression of NADPH oxidase [4, 5]; (2) aldosterone causes an increase in external oxygen free radicals in the peripheral blood [6]; (3) aldosterone produces reactive oxygen species (ROS) by the mitochondrial respiratory chain [7], and (4) aldosterone downregulates the expression of superoxide dismutase and reduces the tissue's ability to produce scavenging oxygen free radicals [8]. AngII and aldosterone also increase inflammatory cytokines, such as interleukin-6 (IL-6) and C-reactive protein, which cause endothelial injury $[9,10]$.

\section{Inflammatory Cytokines}

It has been confirmed that one of the mechanisms of chronic heart failure is an inflammatory reaction. Some inflammatory cytokines can induce myocardial apoptosis and reduce ventricular wall compliance through multiple pathways. When myocardial damage due to acute cardiac insufficiency occurs, neurohormonal and inflammatory cytokines have compensatory effects on the body; however, their persistent activation will cause damage to multiple systems. Activation of the immune response is currently believed to be what causes the generation of inflammatory cytokines. The mechanism may be one of the following: (1) the damaged tissue as an antigen activates an immune reaction $[11,12] ;(2)$ the reduction in an effective output due to various reasons causes prolonged organ ischemia and hypoxia, and then induces the tissue to produce a large number of proinflammatory cytokines; (3) patients with chronic heart failure due to long-term circulation ischemia will experience a release of intestinal endotoxin, which activates the immune response and produces large amounts of inflammatory cytokines [13], and (4) excessive activation of neuroendocrine and autonomic nerve function disorder. Thus far, it has been found that inflammatory cytokines associated with heart failure mechanism include TNF- $\alpha$, IL-6, IL-8, IL-10, IL-1 $\alpha$, IL-1 $\beta$, IL-2, TGF- $\beta$, and IFN- $\gamma$ [14-19]. TNF- $\alpha$ is the main cytokine associated with the mechanism of DHF. Cardiomyocyte apoptosis is induced by TNF- $\alpha$ in the following ways: (1) it activates p38 mitogen-activated protein kinase, triggering apoptosis [20]; (2) it converts nitric oxide (NO) to peroxynitrite ion $\left(\mathrm{ONOO}^{-}\right)$by stimulating iNOS expression, thereby inducing apoptosis of myocardial cells, and (3) it induces myocardial apoptosis by oxidative stress [21].

Various studies have shown that TNF- $\alpha$ can reduce ventricular wall compliance. TNF- $\alpha$ perfusion may cause an increase in left ventricular (LV) end-diastolic volume and impaired myocardial compliance. TNF- $\alpha$ affects MMP activity as well as tissue inhibitor of metalloproteinase (TIMP) activity. The generation of myocardial collagen fibers is associated with MMP/ TIMP activity. In the early inflammatory stage, the MMP activity exceeds TIMP activity; thus, the generation of collagen fibers is reduced and causes LV dilatation. With the development of disease, MMP activity decreases but the level of TIMPs is increased and the level of collagen fibers is decreased [22]. 


\section{The Process of Myocardial Calcium Cycling and Associated Protein}

Myocardial dysfunction is related to the abnormal cycling process of myocardial calcium. The calcium cycle includes cardiac sarcoplasmic reticulum, calcium release, calcium reuptake, and calcium storage [23]. Research shows that the main process of diastolic function is calcium reuptake. Under normal physiological conditions, after the myocardial cell membrane is depolarized, a small amount of intracellular $\mathrm{Ca}^{2+}$ activates the calcium release channel of the sarcoplasmic reticulum to release large amounts of $\mathrm{Ca}^{2+}$ into the cytoplasm, which rapidly increases the concentration of $\mathrm{Ca}^{2+}$ in the myocardial tissue. $\mathrm{Ca}^{2+}$ binds with troponin $\mathrm{C}$ to trigger the sliding filament and causes myocardial cell contraction. SERCA2a then absorbs $\mathrm{Ca}^{2+}$ in the cytoplasm into the sarcoplasmic reticulum through the consumption of energy. At the same time, the sodium-calcium exchanger (NCX) will transport a small amount of $\mathrm{Ca}^{2+}$ into the cell membrane. The intracellular concentration of $\mathrm{Ca}^{2+}$ decreases rapidly, $\mathrm{Ca}^{2+}$ and troponin $\mathrm{C}$ become dissociated, and the cardiomyocytes become relaxed [24].

Research has shown that in the early stage of the disease, through a compensatory role, the expression of SERCA2a is usually upregulated. When serious heart failure occurs, the expression level of SERCA2a decreases markedly [25, 26]. Leszek et al. [27] found that SERCA2a reduces the expression of diastolic protein levels in DHF. SERCA2a activity also has an effect on the uptake of calcium by the sarcoplasmic reticulum. SERCA2a activity is mainly regulated by phospholamban (PLB). PLB is a single transmembrane protein with 52 amino acid residues and is mainly expressed in the sarcoplasmic reticulum of cardiac muscle, smooth muscle, and slow-twitch fibers. Changes in its expression levels and phosphorylation state can directly influence SERCA2a activity [28-34]. However, some researchers believe that PLB phosphorylation status plays a more important role in SERCA2a activity. When PLB is dephosphorylated, it can combine with SERCA2a to depress the calcium pump activity by decreasing the affinity of SERCA2a for $\mathrm{Ca}^{2+}$. In contrast, when it is phosphorylated, its inhibitory effect is eliminated, and the calcium pump activity is increased [35]. Unlike the elevated NCX expression level in systolic heart failure [36], NCX expression is unchanged in DHF, which has no compensation for the rate of decline in intracytoplasm $\mathrm{Ca}^{2+}$ in the diastole. In the diastole, the continuous increase in $\mathrm{Ca}^{2+}$ concentration leads to $\mathrm{Ca}^{2+}$ not dissociating from troponin, causing diastolic myocardial sustained contraction. Thus, the decline in myocardial relaxation is due to the performance of DHF. On the other hand, the increased concentration of $\mathrm{Ca}^{2+}$ in the cytoplasm can activate $\mathrm{Ca}^{2+}$-dependent ATPase in cells, resulting in increased ATP degradation. The process of ATP degradation will produce large amounts of active oxygen, hydrogen peroxide, and superoxide anion. Through a variety of mechanisms, these substances decrease vasodilation, causing myocardial damage and eventually leading to ventricular remodeling.

\section{Degradation of ATP and Oxidative Stress}

Hypoxia and reperfusion can cause degradation of a significant quantity of ATP. Hypoxanthine, xanthine, and uric acid are ATP degradation products. Hypoxanthine needs xanthine oxidase (XO) to be catalyzed into xanthine. $\mathrm{XO}$ is also needed to convert xanthine into uric acid. In the process, it produces ROS, hydrogen peroxide $\left(\mathrm{H}_{2} \mathrm{O}_{2}\right)$, and superoxide anion $\left(\mathrm{O}_{2}{ }^{-}\right)$ [37]. XO is converted from xanthine oxidoreductase. Another transformation product of xanthine oxidoreductase is xanthine dehydrogenase [38], which can be converted to XO by various enzymes. Under hypoxia or reperfusion, a large amount of ATP is degraded and hypoxanthine is produced. The aggregated hypoxanthine increases the substrate for $\mathrm{XO}$, which 
activates the metabolic pathway to convert xanthine dehydrogenase into XO. Some studies have shown that blood vessel endothelia and cardiac muscle can produce XO locally [39]. The effect of $\mathrm{XO}$ on hypoxanthine increases the generation of oxidative radicals and then causes myocardial injury and ventricular remodeling.

Oxygen free radicals are involved in cardiac remodeling in several ways, including inducing protein oxidization, DNA strand breaking, oxidization of cell membrane lipids, activating nuclear factor- $\kappa \mathrm{B}$, and activating mitogen-activated protein kinase [40-42]. For example, oxidized low-density lipoprotein (LDL) is formed because of the action of oxygen free radicals on LDL. LDL initiates the chain reaction of lipid peroxidation, and forms more oxidized LDL. Oxidized LDL damages endothelial cells and their function in a variety of ways, and then promotes oxidative stress, resulting in vasomotor dysfunction [43]. $\mathrm{O}_{2}{ }^{-}$and $\mathrm{NO}$ react to form $\mathrm{ONOO}^{-}$. In addition to lipid peroxidation, this process depletes $\mathrm{NO}$, and then weakens the relaxant effect of NO on blood vessels and, as a result, the hypoxia worsens. Through the activation of MMP, ROS mediate the signal pathway of cardiomyocyte hypertrophy. This causes structural changes in the extracellular matrix, which then induces hypertrophy and apoptosis.

In summary, increases in preload and afterload as well as other factors will cause RAAS activation. Released aldosterone increases type I collagen, which results in oxidative stress and induces elevation of inflammatory cytokines. Injury and hypoxia can induce inflammatory reactions. TNF- $\alpha$ induces apoptosis through the activation of associated protein and oxidative stress. The changes in collagen fibers affected by TNF- $\alpha$ cause a decline in myocardial compliance. ATP is reduced due to hypoxia and ischemia. PLB phosphorylation is inhibited, and SERCA2a activity regulated by PLB is decreased, which causes a decline in the ability of sarcoplasmic reticulum calcium uptake. The elevated $\mathrm{Ca}^{2+}$ concentration leads to cardiac spasm and an increase in the degradation of ATP. Active oxygen and oxidizing material produced in the process of ATP decomposition promote oxidative stress and induce hypertrophy and apoptosis directly. Any one of these four mechanisms can be the initial mechanism of DHF onset and then activate another mechanism. The final result is ventricular reconstruction and a decrease in ventricular wall compliance, which is the most direct cause of DHF. The indication of molecular mechanism of heart failure might provide integrative understanding and increase the reserve ability of heart problems [44].

\section{Acknowledgments}

This work was supported by The National High Technology Research and Development Program of China (863 Program, 2010AA0221396001) and Shanghai Science and Technology Committee (13401904400; 12401903400).

\section{Disclosure Statement}

The authors declare that there are no conflicts of interest regarding the publication of this article. 


\section{References}

1 Fuster V, Alexander RW, Orourke RA: Hurst Cardiology. Beijing, People’s Medical Publishing House, 2008, p 670.

2 Gerdes AM: Cardiac myocyte remodeling in hypertrophy and progression to failure. J Card Fail 2002;8:S264S268.

3 Calderone A, Takahashi N, Izzo NJ, et al: Pressure- and volume-induced left ventricular hypertrophies are associated with distinct myocyte phenotypes and differential induction of peptide growth factor mRNAs. Circulation 1995; 92:2385-2390.

4 Johar S, Cave AC, Narayanapanicker A, et al: Aldosterone mediates angiotensin II-induced interstitial cardiac fibrosis via a Nox2-containing NADPH oxidase. FASEB J 2006;20:1546-1548.

5 Park YM, Park MY, Suh YL, et al: NADPH oxidase inhibitor prevents blood pressure elevation and cardiovascular hypertrophy in aldosterone-infused rats. Biochem Biophys Res Commun 2004,313:812-817.

6 Ahokas RA, Warrington KJ, Gerling IC, et al: Aldosteronism and peripheral blood mononuclear cell activation: a neuroendocrine-immune interface. Circ Res 2003;93:e123-e135.

7 Zhang A, Jia Z, Guo X, et al: Aldosterone induces epithelial-mesenchymal transition via ROS of mitochondrial origin. Am J Physiol Renal Physiol 2007;293:F723-F731.

8 Onozato Ml, Tojo A, Kohayashi N, et al: Dual blockade of aldosterone and angiotensin II additively suppresses TGF-beta and NADPH oxidase in the hypertensive kidney. Nephrol Dial Transplant 2007;22:1314-1322.

9 Luther JM, Gainer JV, Murphey LJ, et al: Angiotensin II induces interleukin-6 in humans through a mineralocorticoid receptor-dependent mechanism. Hypertension 2006;48:1050-1057.

10 Kotlyar E, Vita JA, Winter MR, et al: The relationship between aldosterone, oxidative stress, and inflammation in chronic, stable human heart failure. J Card Fail 2006;12:122-127.

11 Nian ML, et al: Inflammatory cytokines and postmyocardial infarction remodeling. Circ Res 2004;94:15431553.

12 Li M, et al: p38 MAP kinase mediates inflammatory cytokine induction in cardiomyocytes and extracellular matrix remodeling in heart. Circulation 2005;111:2494-2502.

13 Peschel T, et al: Invasive assessment of bacterial endotoxin and inflammatory cytokines in patients with acute heart failure. Eur J Heart Fail 2003;5:609-614.

14 Van Tassell BW, et al: Enhanced interleukin-1 activity contributes to exercise intolerance in patients with systolic heart failure. PLoS One 2012;7:433-438.

15 Haugen E, et al: Increased interleukin- 6 but not tumour necrosis factor-alpha predicts mortality in the population of elderly heart failure patients. Exp Clin Cardiol 2008;13:19-24.

16 Helwig BG, et al: Increased interleukin-6 receptor expression in the paraventricular nucleus of rats with heart failure. Am J Physiol Regul Integr Comp Physiol 2007;292:R1165-R1173.

17 Sikkeland LI, et al: Increased levels of inflammatory cytokines and endothelin-1 in alveolar macrophages from patients with chronic heart failure. PLoS One 2012;7:e36815.

18 Boffa GM, et al: Interleukin-6 and tumor necrosis factor-alpha as biochemical markers of heart failure: a headto-head clinical comparison with B-type natriuretic peptide. J Cardiovasc Med (Hagerstown) 2009;10:758764.

19 Gullestad L, et al: Inflammatory cytokines in heart failure: mediators and markers. Cardiology 2012;122: 23-35.

20 Prickett TD, Brautigan DL: Cytokine activation of p38 mitogen-activated protein kinase and apoptosis is opposed by alpha- 4 targeting of protein phosphatase 2A for site-specific dephosphorylation of MEK3. Mol Cell Biol 2007;27:4217-4227.

21 Haudek SB, et al: TNF provokes cardiomyocyte apoptosis and cardiac remodeling through activation of multiple cell death pathways. J Clin Invest 2007;117:2692-2701.

22 Sivasubramanian N, et al: Left ventricular remodeling in transgenic mice with cardiac restricted overexpression of tumor necrosis factor. Circulation 2001;104:826-831.

23 Dibb KM, Graham HK, Venetucci LA, et al: Analysis of cellular calcium fluxes in cardiac muscle to understand calcium homeostasis in the heart. Cell Calcium 2007;42:503-512.

24 Bers DM: Cardiac excitation-contraction coupling. Nature 2002;415:198-205.

25 van der Velden J, Merkus D, Klarenbeek BR, et al: Alterations in myofilament function contribute to left ventricular dysfunction in pigs early after myocardial infarction. Circ Res 2004; 95:e85-e95.

26 Zarain Herzberg A: Regulation of the sarcoplasmic reticulum $\mathrm{Ca}^{2+}$-ATPase expression in the hypertrophic and failing heart. Can J Physiol Pharmacol 2006;84:509-521.

27 Leszek P, Szperl M, Klisiewicz A, et al: Alterations in calcium regulatory protein expression in patients with preserved left ventricle systolic function and mitral valve stenosis. J Card Fail 2008;14:873-880.

28 MacLennan DH, Kranias EG: Phospholamban: a crucial regulator of cardiac contractility. Nat Rev Mol Cell Biol 2003;4:566-577.

29 MacLennan DH, Asahi M, Tupling AR: The regulation of SERCA-type pumps by phospholamban and sarcolipin. Ann NY Acad Sci 2003;986:472-480.

30 MacLennan DH, Abu-Abed M, Kang C: Structure-function relationships in $\mathrm{Ca}^{2+}$ cycling proteins. J Mol Cell Cardiol 2002;34:897-918. 
31 Bhupathy P, Babu GJ, Periasamy M: Sarcolipin and phospholamban as regulators of cardiac sarcoplasmic reticulum $\mathrm{Ca}^{2+}$ ATPase. J Mol Cell Cardiol 2007;42:903-911.

32 Brittsan AG, Kranias EG: Phospholamban and cardiac contractile function. J Mol Cell Cardiol 2000;32:21312139.

33 Frank K, Kranias EG: Phospholamban and cardiac contractility. Ann Med 2000;32:572-578.

34 Vangheluwe P, Schuermans M, Zador E, et al: Sarcolipin and phospholamban mRNA and protein expression in cardiac and skeletal muscle of different species. Biochem J 2005;389:151-159.

35 Nef HM, Mollmann H, Skwara W, et al: Reduced sarcoplasmic reticulum $\mathrm{Ca}^{2+}$-ATPase activity and dephosphorylated phospholamban contribute to contractile dysfunction in human hibernating myocardium. Mol Cell Biochem 2006;282:53-63.

36 Conway SJ, Koushik SV: Cardiac sodium-calcium exchanger: a double-edged sword l. Cardiovasc Res 2001;52: 194-197.

37 Chao IN, Zhu W: Drug treatment of chronic congestive heart failure. Basic Med Sci Clin 2002;22:26-31.

38 Pfeffer M, Braunwald E, Moyé LA, et al; the SAVE Investigators: Effect of captopril on mortality and morbidity in patients with left ventricular dysfunction after myocardial infarction: results of the survival and ventricular enlargement trial. N Engl J Med 1992;327:669-677.

39 Konstam MA, Rousseau MF, Kronenberg MW, et al: Effects of the angiotensin converting enzyme inhibitor enalapril on the long-term progression of left ventricular dysfunction in patients with heart failure. SOLVD Investigator. Circulation 1992;86:431-438.

40 Kobayashi N, Yoshida K, Nakano S, et al: Cardioprotective mechanisms of eplerenone on cardiac performance and remodeling in failing rat hearts. Hypertension 2006;47:671-679.

41 Gupta S, Young D, Sen S: Inhibition of NF-kB induces regression of cardiac hypertrophy, independent of blood pressure control, in spontaneously hypertensive rats. Am J Physiol Heart Circ Physiol 2005;289:H20-H29.

42 Rude KM, Duhaney ST, Kuster MG, et al: Aldosterone stimulates matrix metalloproteinases and reactive oxygen species in adult rat ventricular cardiomyocytes. Hypertension 2005;46:555-561.

43 Van Kraaij DJW, Jansen RWMM, Bouwels LHR, et al: Furosemide withdrawal in elderly heart failure patients with preserved left ventricular systolic function. Am J Cardiol 2000;85:1461-1466.

44 Pan W, Zhou H: Inclusion of integrative medicine in clinical practice. Integr Med Int 2014;1:1-4. 